\title{
Concerto
}

\section{Urs Stoffel}

Dr med., membre du Comité central de la FMH, responsable a.i. du département Tarifs et conventions pour la médecine ambulatoire en Suisse

\section{Contexte}

En 2010, la FMH - alors encore seule - a lancé l'ambitieux projet de révision globale de la structure tarifaire TARMED sous le nom de TARVISION. Avant 2010, des tentatives avaient déjà eu lieu dans le cadre de la société simple «TARMED Suisse», regroupant les fournisseurs de prestations et les assureurs depuis l'introduction du TARMED, afin de réviser la structure tarifaire. Mais les efforts consentis se sont tous heurtés au droit de veto des différents partenaires, étant donné que la prise de décision de TARMED Suisse reposait sur le principe de l'unanimité.

Plus de 26 équipes spécialisées et plus de 150 personnes travaillent activement sur les différents chapitres de la révision.

En 2012, la FMH est parvenue à mettre sur pied un partenariat tripartite aux côtés de l'association des hôpitaux $\mathrm{H}+$ et de la Commission des tarifs médicaux LAA CTM afin d'entreprendre ensemble la refonte totale de la structure tarifaire du TARMED. Cette révision représente un énorme défi sachant que ce tarif à la prestation fort de plus de 4700 positions n'a pratiquement jamais été adapté aux conditions actuelles depuis son introduction en 2004.

Depuis, la FMH et ses partenaires travaillent d'arrachepied à cette révision. Pour tous les participants, il était clair dès le départ qu'il fallait impérativement séparer la structure tarifaire du prix des prestations. C'est en effet le seul moyen d'obtenir une structure tarifaire appropriée, conforme aux règles de l'économie d'entreprise comme l'exige la LAMal et adaptée aux évolutions actuelles. Entretemps, l'association fầtière curafutura, qui réunit plusieurs assureurs, s'est également ralliée au projet de révision à titre d'observateur. Une fois la nouvelle structure définie, curafutura décidera si elle souhaite cosigner la structure tarifaire révisée qui sera soumise au Conseil fédéral.

A l'heure actuelle, plus de 26 équipes spécialisées et plus de 150 personnes travaillent activement sur les différents chapitres de la révision avec les experts des sociétés médicales de la FMH. Chaque équipe est dirigée par un représentant des partenaires de la révision, et chapeautée par une société de discipline qui met à dis- position ses délégués tarifaires pour réviser la nomenclature à titre d'experts avec la direction de l'équipe. L'objectif visé consiste à présenter une proposition commune et consensuelle pour chaque chapitre. $\mathrm{Au}$ terme de la révision, les différents chapitres seront mis en consultation à l'interne auprès de toutes les sociétés médicales de la FMH.

\section{Conditions-cadres contraignantes avec des dispositions conformes à la loi pour un tarif applicable}

Au cours de la révision, les conditions-cadres et les dispositions légales relatives à la mise en œuvre de la révision ont régulièrement fait l'objet de discussions. Dans plusieurs courriers à la FMH et aux partenaires tarifaires, de même que dans sa réponse à l'interpellation du conseiller national Thomas Weibel sur la révision du TARMED [1], le Conseil fédéral a défendu une position claire sur les dispositions et conditions-cadres de la révision tarifaire.

Le Conseil fédéral exige une double neutralité des coûts au sens d'une neutralité statique pour la révision de la structure et d'une neutralité dynamique pour le prix qui doit, le cas échéant, compenser l'évolution du volume de prestations à l'aide d'un mécanisme de correction, ce qui revient de facto à définir un budget global. En outre, le Conseil fédéral n'accepte aucune indexation des coûts et exige que la structure tarifaire «[repose] sur de nouveaux relevés

\section{Cela revient de facto à définir un budget global.}

des données relatives aux coûts et aux prestations et servant de base au modèle tarifaire». Dans sa réponse à l'interpellation du CN Weibel, il précise ce qui suit au point 4:

«Si, au final, l'augmentation des coûts s'avère inévitable pour l'AOS (en raison, par ex., d'un traitement de meilleure qualité ou d'une variation des coûts de revient), celle-ci doit être très limitée en raison de la viabilité financière de l'ensemble du système (principe d'équité). Dans les décisions qu'il a prises jusqu'ici, le Conseil fédéral a toujours estimé que la viabilité financière de l'ensemble du système doit en 


\section{Congrès sur les tarifs}

Congrès: Tarife in der Sozialversicherung - Zwischen Norm und Vertrag: Interdisziplinärer Blick auf Grundlagen - Gestaltungskriterien - Entwicklungen

Jeudi 19 novembre 2015, Eventforum Berne, 13 h30 à 17h30. Avec Andreas Bührer, Dr sc. nat. et med.; Ruth Humbel, lic. iur., conseillère nationale; Ueli Kieser, Prof. iur., avocat; Stefan Meierhans, Dr iur., Surveillant des prix; Willy Oggier, Dr oec. HSG, économiste de la santé; Oliver Peters, lic. rer. pol., vicedirecteur de l'Office fédéral de la santé publique; et Urs Stoffel, Dr med., membre du Comité central de la FMH. Veuillez vous inscrire en ligne à l'adresse www.irp.unisg.ch, par courriel à irp[at]unisg.ch ou par la poste à: Institut de sciences juridiques et de la pratique juridique (IRP-HSG), Bodanstrasse 4, 9000 St-Gall.

principe primer sur la justification du tarif du point de vue de l'économie d'entreprise.» [1]

Au vu des précisions du Conseil fédéral et du débat controversé entre les fournisseurs de prestations et les assureurs sur les critères de la structure tarifaire, la FMH a décidé de lancer le projet "Concerto".

\section{Quelles sont les dispositions prévues par la} LAMal pour garantir une structure et un ajustement tarifaires conformes à la loi et appropriés?

\section{Quel est l'objectif du projet Concerto?}

Comme une structure tarifaire dépend de différents facteurs et paramètres, la FMH a décidé de soumettre la question suivante à trois experts reconnus dans le domaine des modèles tarifaires, de l'économie de la santé et du droit: quelles sont les conditions-cadres et les dispositions prévues par la LAMal pour garantir une structure et un ajustement tarifaires conformes à la loi et appropriés? Chacun de ces trois experts a été invité à mettre en évidence les dispositions qui, à son sens, doivent impérativement être respectées pour que le tarif puisse être accepté par toutes les parties.
Andreas Bührer, Dr sc. nat. et med., s'est penché sur les modèles tarifaires et la méthode sur laquelle repose le tarif; Willy Oggier, Dr oec. HSG, s'est intéressé aux aspects économiques du tarif et de la révision tarifaire; et Ueli Kieser, Prof. iur., s'est quant à lui concentré sur les dispositions légales et juridiques du tarif et de la révision. Pour que le tarif puisse être appliqué au quotidien, il doit répondre aux aspects spécifiques à chaque discipline mais il est tout aussi important qu'il intègre les aspects énoncés plus haut, à savoir la méthode tarifaire, les aspects économiques et les dispositions légales.

Dans les trois articles suivants, vous trouverez un résumé des travaux des trois experts qui se sont penchés sur le tarif et la révision tarifaire. La version intégrale de ces articles sera publiée à la mi-octobre dans un ouvrage intitulé Tarif und Tarifanpassung in der Krankenversicherung - Ein juristischer, ökonomischer und methodischer Blick auf den ambulanten Bereich (Editions Stämpfli; en allemand avec résumés en français, N.D.T.). Enfin, un symposium et un débat organisés par l'Institut de sciences juridiques et de pratique juridique de l'Université de Saint-Gall se tiendront le 19 novembre 2015 à l'Eventforum de Berne sous le titre «Tarife in der Sozialversicherung - Zwischen Norm und Vertrag».

\section{Références}

1 Curia Vista. 15.3182 - Interpellation «Tarmed. Révision de la structure tarifaire» du CN Thomas Weibel (pvl ZH) du 18.3.2015.

\section{Tarif et adaptation tarifaire dans I'assurance-maladie}

Les résultats des trois experts sont disponibles dans un ouvrage paru en octobre 2015. Cette publication de 200 pages coûte 50 francs et peut être commandée auprès des Editions Stämpfli. Vous trouverez de plus amples informations sous: www.staempfliverlag.com 